\title{
Effect of temperature and $\alpha$-irradiation on gas permeability for polymeric membrane
}

\author{
VAIBHAV KULSHRESTHA, K AWASTHI, N K ACHARYA, M SINGH and Y K VIJAY* \\ Department of Physics, University of Rajasthan, Jaipur 302 004, India
}

MS received 22 April 2005; revised 21 September 2005

\begin{abstract}
In the present study the polyethersulphone (PES) membranes of thickness $(35 \pm 2) \mu \mathrm{m}$ were prepared by solution cast method. The permeability of these membranes was calculated by varying the temperature and by irradiation of $\alpha$ ions. For the variation of temperature, the gas permeation cell was dipped in a constant temperature water bath in the temperature range from 303-373 K, which is well below the glass transition temperature $(498 \mathrm{~K})$. The permeability of $\mathrm{H}_{2}$ and $\mathrm{CO}_{2}$ increased with increasing temperature. The PES membrane was exposed by $\alpha$-source $\left({ }_{95} \mathrm{Am}^{241}\right)$ of strength $(1 \mu \mathrm{Ci})$ in vacuum of the order of $10^{-6}$ torr, with fluence $2.7 \times 10^{7}$ ions $/ \mathrm{cm}^{2}$. The permeability of $\mathrm{H}_{2}$ and $\mathrm{CO}_{2}$ has been observed for irradiated membrane with increasing etching time. The permeability increases with increasing etching time for both gases. There was a sudden change in permeability for both the gases when observed at 18 min etching. At this stage the tracks are visible with optical instrument, which confirms that the pores are generated. Most of pores seen in the micrograph are circular cross-section ones.
\end{abstract}

Keywords. Activation energy; gas permeability; permselectivity; track etched membrane; critical etching time.

\section{Introduction}

The development of polymeric membranes for gas separation has provided an alternative to the traditional energy-intensive processes. Gas separation became a major industrial application of membrane technology. For example, the commercial use of polymer membranes for air separation, the recovery of hydrogen from nitrogen, carbon monoxide and methane mixtures, and the removal of carbon dioxide from natural gas has been widely reported (Stern 1994). For the industrial use, high permeability and excellent selectivities have relevance on glassy polymer membranes that help separate gas. In addition to the chemical composition, the transport properties are related to the main characteristics of copolymers like the glass transition temperature, crystallinity and crosslinking ratio. Gas diffusion through polymers is related to the activation energy (Pesiri et al 2003). Gas diffusion coefficients typically increase appreciably with increasing temperature when the polymer does not undergo thermally induced morphological rearrangements such as crystallization over the temperature range of interest (Ghosal and Freeman 1993), since both diffusivity and solubility coefficients are temperature dependent.

Membranes possessing negligible permeability at room temperature may exhibit enhanced performance at elevated temperature as increase in diffusivity results in de-

*Author for correspondence (yk_vijay@sancharnet.in) crease in solubility. Changing the operating temperature of a membrane can be used to tune and enhance overall performance by means of its permeability (Acharya et al 2004b).

Latent tracks provide enhanced diffusion, whereby the permeability depends strongly on the track recording material and on the types of the transmitted substance. Track-etch membranes offer distinct advantages over conventional membranes due to their precisely determined structure. Their pore size, shape and density can be varied in a controllable manner so that a membrane with the required transport and retention characteristics can be produced (Fleischer et al 1975; Avasthi et al 1998; Apel 2003). In ion irradiated polymeric membrane the trail of damaged material that are left along the ion trajectories can be preferentially etched to open and enlarge, which is the basis for the wide use of polymeric membrane as membrane filters. In the present work, we have measured permeability of hydrogen and carbon dioxide for polyethersulphone membranes at different temperatures and using irradiation of $\alpha$ particles.

\section{Experimental}

\subsection{Sample preparation}

Polyethersulphone (PES) was obtained in granular form from Gadra Chemicals, Bharuch. The membranes of thickness, $35( \pm 2) \mu \mathrm{m}$, were prepared by solution cast method. The details of solution cast method have been 
given elsewhere (Vijay et al 2002). The membranes were dried in low vacuum at $60^{\circ} \mathrm{C}$ for $24 \mathrm{~h}$ to completely remove the solvent.

\subsection{Irradiation}

The polyethersulphone membrane was exposed by $\alpha$-source $\left({ }_{95} \mathrm{Am}^{241}\right.$ ) of strength, $1 \mu \mathrm{Ci}$, having energy, $5 \cdot 3 \mathrm{MeV}$, kept at a distance of $2.5 \mathrm{~cm}$ for irradiation in vacuum of the order of $10^{-6}$ torr. The initial dose was measured using GM Counter and was found to be 300 particles per second. The dose of $2.7 \times 10^{7}$ ions $/ \mathrm{cm}^{2}$ was obtained in $23 \mathrm{~h}$. Schematic of the irradiation of polymer membrane is shown in figure 1.

\subsection{Chemical etching}

The irradiated membranes were etched chemically in $6 \mathrm{~N}$ $\mathrm{NaOH}$ at $60 \pm 2{ }^{\circ} \mathrm{C}$ (Barbari and Koros 1998). The etching time was increased with the step of 1 min and after every etching the membranes were washed thoroughly by distilled water. The etchant changed periodically so that concentration of etchant remained the same during experiment and the temperature of etchant was also kept constant throughout etching.

\subsection{Gas permeability measurements and optical micrographs}

Permeability is defined as the product of solubility and diffusivity coefficient and obeys Fick's law. The flow rate was measured using permeability cell and calculated the permeability using Fick's formula as discussed by Acharya et al (2004a) and Liu et al (2001), whereas the permselectivity of a polymeric membrane for one gas over another is given by the ratio of their permeability.

In the present work we have measured the effect of temperature and $\alpha$ irradiation with subsequent chemical etching, on the gas permeability for the membranes. The temperature varies from $303 \mathrm{~K}$ to $373 \mathrm{~K}$, the measuring range of temperature is selected such that the physical state of polymer does not change. The electron microscopic grid has a circular dimension having $3 \mathrm{~mm}$ diameter. There are 1000 very small squares in a small area. This is used as a reference scale. The side of a small square is magnified under LABOMED $(\times 10)$ microscope at various magnifications i.e. 10,40 and 100 . The micrographs are stored in computer through standard software (PixelView).

\section{Results and discussion}

\subsection{Effect of temperature on gas permeability}

The increased segmental motion at higher temperatures undermines the ability of polymer to discriminate between penetrants of different physical dimensions, thereby resulting in loss in diffusivity. The temperature changes also affect the solubility, which is governed primarily by the chemical nature of the penetrant and polymer-penetrant interactions. For most gases, as temperature increases, the solubilities increase (Costello and Koros 1994).

\section{Polymer Membrane}

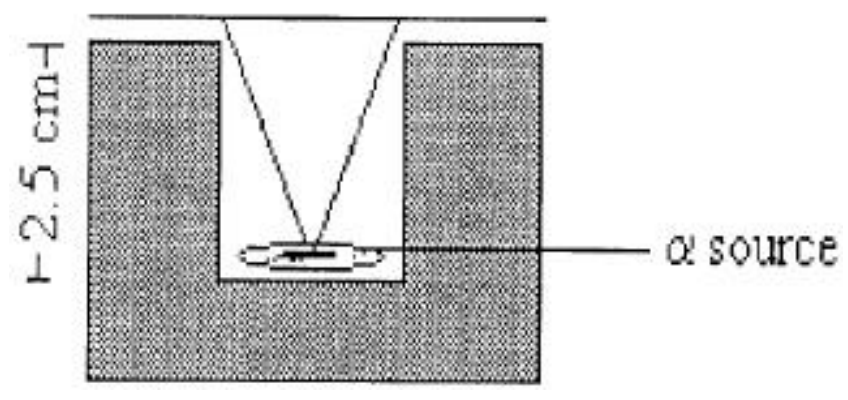

Figure 1. Schematic of irradiation of polymer membrane.
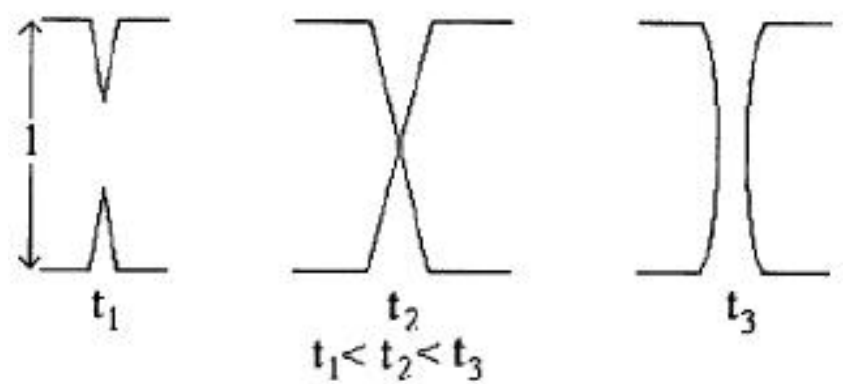

Figure 2. Schematic diagram of track formation with etching time.

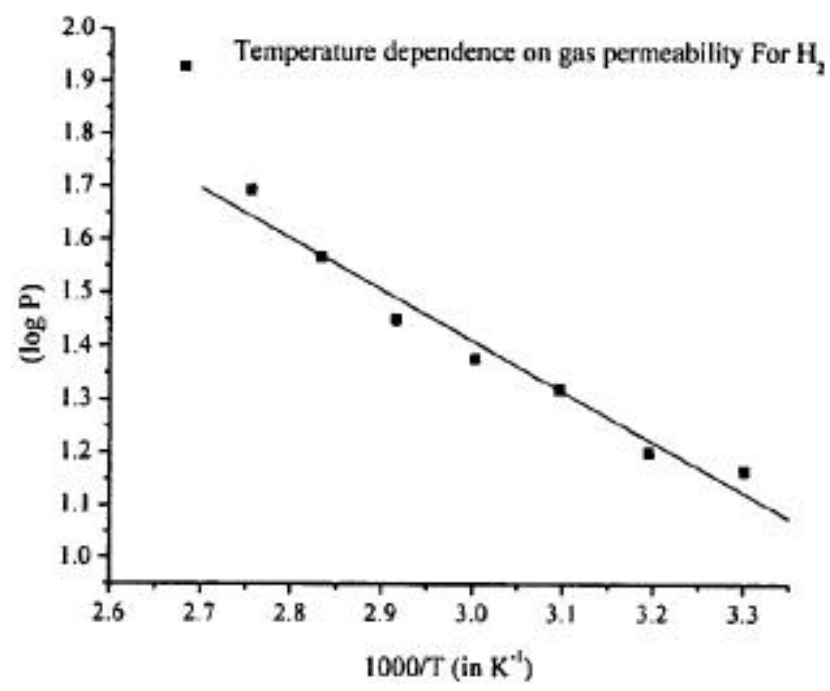

Figure 3. $\log P$ vs $1000 / T$. 
Permeation is an activated process and the permeability coefficient can be approximated by an Arrhenius expression. Figure 3 shows the temperature dependence on permeation, curve between $\log P$ vs $1 / T$ gives a straight line, which is in accordance with Arrhenius equation and determine the activation energy for permeation.

The permeability of membrane does not depend on pressure of gas at lower pressure range (Acharya et al 2004b). The solubility of both the gases in polymer in given pressure range is almost the same, but temperature affects the diffusion process. Figure 3 shows that the permeability increases with temperature due to the change in diffusivity of gas in the polymer matrix. The activation energy for permeation of hydrogen is calculated and found to be $7.91 \mathrm{~kJ} / \mathrm{mol}$, which is almost equal to the activation energy for permeation of carbon dioxide $(7.72 \mathrm{~kJ} / \mathrm{mol})$. This is due to the structure of the polymer matrix. Acharya et al (2004b) studied the temperature dependent gas permeability for polycarbonate membrane, and activation energy for permeation of hydrogen is $5.5 \mathrm{~kJ} / \mathrm{mol}$, whereas in case of carbon dioxide it is $11.5 \mathrm{~kJ} / \mathrm{mol}$, due to its low glass transition temperature (Acharya et al 2004b).

\subsection{Effect of irradiation on gas permeability}

The permeability of irradiated membrane depends on operating conditions such as temperature, pressure and composition as well as on structural features of the materials. The permeability increases with etching time and at a particular etching time a large enhancement in permeability was observed, called critical etching time (Vijay et al 2003). It depends on irradiant parameters like size, mass, energy and dose of irradiant. In polymers, the permeability decreases as the permeate size increases. The permeability for hydrogen and carbon dioxide has been measured for etched membrane.

The measurements have been performed for $\alpha$-irradiated and track etched membranes. The $\alpha$-irradiated polycarbonate (PC) also shows similar measurement (Vijay et al 2003), but the critical etching time for PC is more than the present case. It may be concluded that the electronic energy loss $(\mathrm{d} E / \mathrm{d} X)_{\mathrm{e}}$ for PC is less than PES. The permeability of the membrane increases gradually with etching, but at the critical etching time permeability increases rapidly, which is about $18 \mathrm{~min}$ as given in figure 4. It is expected from the observation that at critical etching time conically etched tracks from both sides of membrane are just meeting and opening as shown in figure 2 at time $t_{2}$, this type of track helps in the separation of gases of different molecular sizes. In order to study the effect of molecular size on permeability, measurements for $\mathrm{H}_{2}$ and $\mathrm{CO}_{2}$ gases have been performed at the same temperature and pressure. The $\mathrm{CO}_{2}$ molecules are larger than $\mathrm{H}_{2}$ molecules, so less flow rate and correspondingly lower permeability was observed. The size of hydrogen molecules is $0.23 \mathrm{~nm}$, whereas size of carbon dioxide molecules is $0.4 \mathrm{~nm}$ (Vijay 2003). By increasing the etching time, the permeability of both the gases, $\mathrm{H}_{2}$ and $\mathrm{CO}_{2}$, increases. The permselectivity also increases with etching time and was found to reach a maximum at the critical etching stage as shown in figure 5. On further etching, the selectivity reduces because gas passes through pore channels that allow passing gases drastically. At this time, size of the track opening goes to higher than the size of the gas molecule as shown in figure 2 at time $t_{3}$ and track goes to a cylindrical shape. The permeability coefficient of any gas for membrane is directly related to the size and shape of tracks. As gases occupy maximum space inside the track, the more and more permeability coefficient achieves, thus we can say permeability coefficient indirectly relates with the etching. Optical micrographs of the surface of unirradiated and chemically etched PES samples at 100 magnification are shown in figure 6 . The effects of irradiation and chemical etching are visible, on the surface of

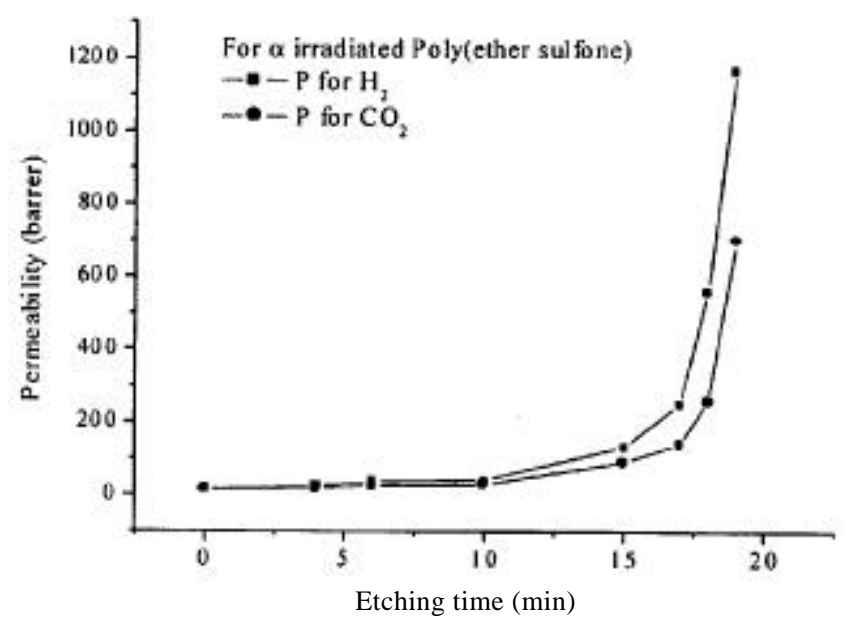

Figure 4. Permeability vs etching time.

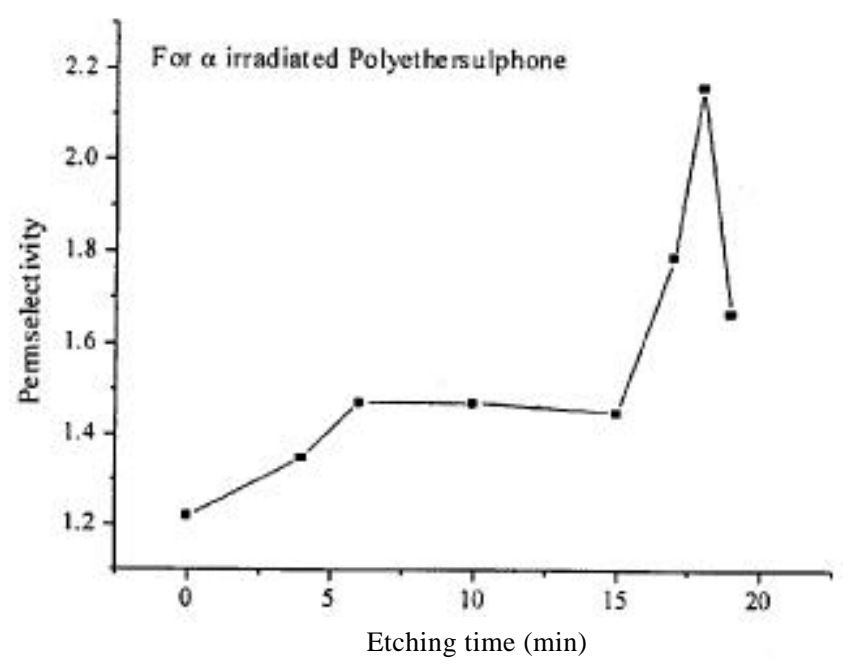

Figure 5. Permselectivity vs etching time. 

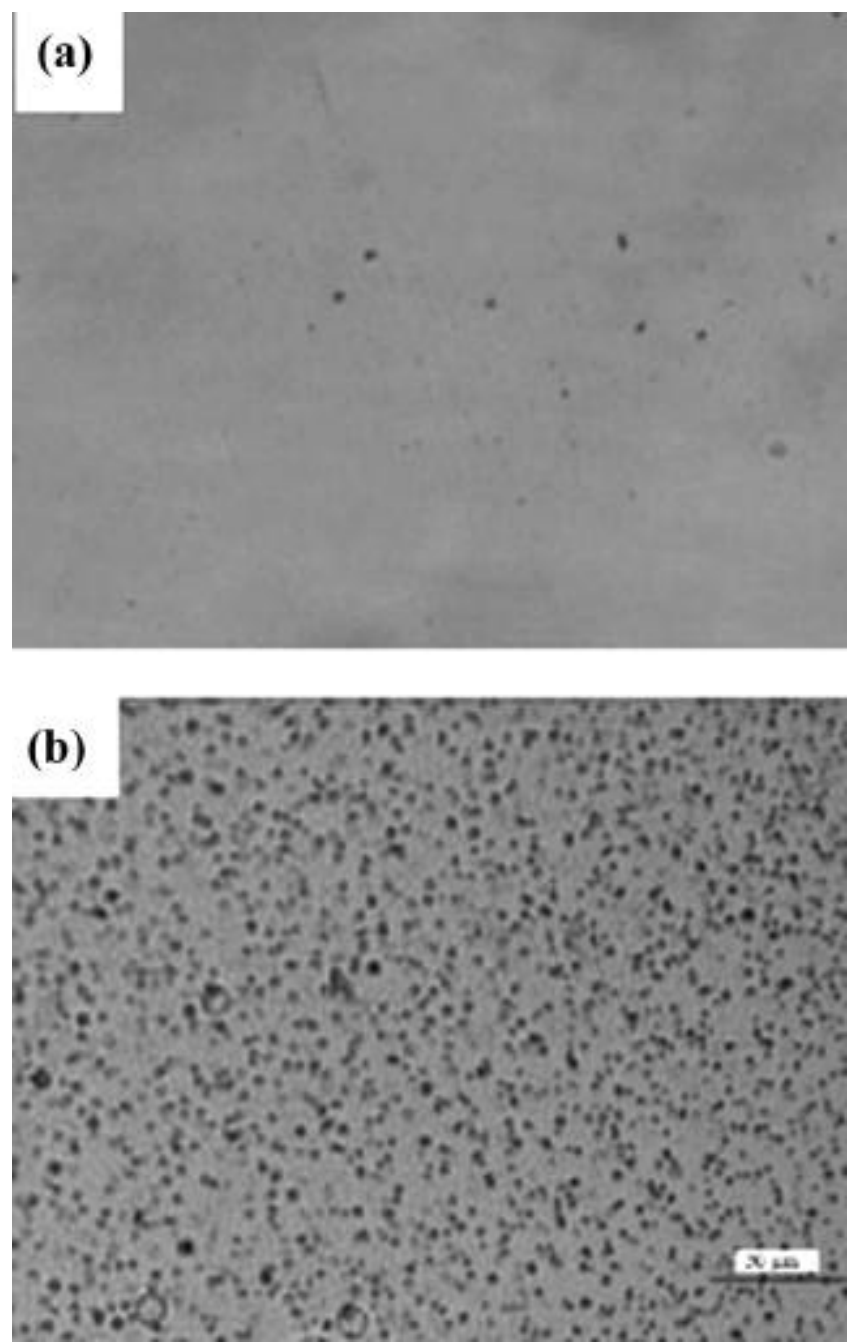

Figure 6. Optical micrographs of (a) unirradiated and (b) irradiated and etched, PES membranes.

the membrane as the circular tracks in figure $6(\mathrm{~b})$. The micrograph supports the gas permeability data.

\section{Conclusions}

The activation energy for permeation of hydrogen and carbon dioxide is almost same, due to the structural feature of polymers. Ion irradiation and subsequent chemical etching is better alternative to modify the permeability of polymeric membranes. The permeability and permselectivity for $\mathrm{H}_{2}$ over $\mathrm{CO}_{2}$ increases up to critical etching time. On further etching permeability increases but permselectivity decreases. Consequently the permeability of hydrogen is greater than that of carbon dioxide.

\section{Acknowledgements}

Authors are grateful to Gadra Chemicals for providing the polymer for preparing the membrane samples. One of the authors (VK) is thankful to Mr Vibhav K Saraswat, Vimal Kishor and Ankur Jain for fruitful discussion. Authors are thankful to the Nuclear Science Centre, New Delhi and Ministry of Non-Conventional Energy Sources, New Delhi, for providing financial assistance for the work.

\section{References}

Acharya N K, Yadav P K, Wate S, Vijay Y K, Singh F and Avasthi D K 2004a Bull. Mater. Sci. 27417

Acharya N K, Yadav P K and Vijay Y K 2004b Indian J. Pure \& Appl. Phys. 42179

Apel P 2003 Nucl. Instrum. \& Meth. Phys. Res. B208 11

Avasthi D K, Singh J P, Biswas A and Bose S K 1998 Nucl. Instrum. \& Meth. Phys. Res. B146 504

Barbari T A and Koros W J 1998 J. Polym. Sci. Part B: Polym. Phys. 26709

Costello L M and Koros W J 1994 J. Polym. Sci. Part B: Polym. Phys. 32701

Fleischer R L, Price P B and Walker R M 1975 Nuclear tracks in solids: Principles and applications (Berkeley: University of California Press)

Ghosal K and Freeman B D 1993 Polym. Adv. Technol. 5673

Liu Ye, Wang Rong and Chung Tai-Shung 2001 J. Membrane Sci. 189231

Pesiri D R, Jorgensen B and Dye R C 2003 J. Membrane Sci. 21811

Stern S A 1994 J. Membrane Sci. 941

Vijay Y K 2003 Radiat. Meas. 3657

Vijay Y K, Wate S, Acharya N K and Garg J C 2002 Int. J. Hydrogen Energy 27905

Vijay Y K, Acharya N K, Wate S and Avasthi D K 2003 Int. J. Hydrogen Energy 281015 